\title{
Stability of Coulomb crystals in a linear Paul trap with storage-ring-like confinement
}

\author{
Kjærgaard, Niels; Mølhave, Kristian; Drewsen, Michael
}

Published in:

Physical Review E. Statistical, Nonlinear, and Soft Matter Physics

Link to article, DOI:

10.1103/PhysRevE.66.015401

Publication date:

2002

Document Version

Publisher's PDF, also known as Version of record

Link back to DTU Orbit

Citation (APA):

Kjærgaard, N., Mølhave, K., \& Drewsen, M. (2002). Stability of Coulomb crystals in a linear Paul trap with storage-ring-like confinement. Physical Review E. Statistical, Nonlinear, and Soft Matter Physics, 66(1), 015401. https://doi.org/10.1103/PhysRevE.66.015401

\section{General rights}

Copyright and moral rights for the publications made accessible in the public portal are retained by the authors and/or other copyright owners and it is a condition of accessing publications that users recognise and abide by the legal requirements associated with these rights.

- Users may download and print one copy of any publication from the public portal for the purpose of private study or research.

- You may not further distribute the material or use it for any profit-making activity or commercial gain

- You may freely distribute the URL identifying the publication in the public portal 


\title{
Stability of Coulomb crystals in a linear Paul trap with storage-ring-like confinement
}

\author{
Niels Kjærgaard, ${ }^{1, *}$ Kristian Mølhave, ${ }^{2, \dagger}$ and Michael Drewsen ${ }^{3, \sharp}$ \\ ${ }^{1}$ Optics and Fluid Dynamics Department, Rish National Laboratory, DK-4000 Roskilde, Denmark \\ ${ }^{2}$ Mikroelektronik Centret, Technical University of Denmark, DK-2800 Kgs. Lyngby, Denmark \\ ${ }^{3}$ Institute of Physics and Astronomy, University of Aarhus, DK-8000 Aarhus C, Denmark
}

(Received 9 April 2002; published 23 July 2002)

\begin{abstract}
We report experiments on the stability of ion Coulomb crystals in a linear Paul trap with storage-ring-like confinement. The transverse dynamics of charged particles in a trap of this type is analogous to that of a fast beam traveling through a channel with periodic, magnetic alternating gradient confinement. The experimentally observed stability conditions for stationary crystals comply remarkably well with current theory of crystalline plasmas and beams.
\end{abstract}

DOI: 10.1103/PhysRevE.66.015401

PACS number(s): 52.27.Lw, 52.27.Jt

During the past decade, extensive theoretical work has been carried out to determine under which conditions spatially ordered states of ion beams, so-called Coulomb crystals, can be attained in storage rings with alternating gradient (AG) focusing [1]. Since a crystalline beam represents the ultimate phase-space density, it is of great interest in beam physics and may find application as a high-luminosity source for, e.g., high-resolution nuclear physics experiments, colliders, and inertial confinement fusion [2]. Real beam experiments on laser-cooled ions in magnetic storage rings have not succeeded in attaining a crystalline ion beam so far [2,3]. However, ordering in a string of ions has been reported in both large-scale magnetic storage rings using electron cooling $[4,5]$ and a small-scale electric storage ring using laser cooling [6]. For the more complex three-dimensional (3D) crystalline ion beams with bulk structures, an essential criterion is that the ring lattice periodicity should be at least $2 \sqrt{2}$ times as high as the maximum betatron (bare) tune $[7,8]-\mathrm{a}$ criterion which hitherto has not been fulfilled in the magnetic storage ring experiments on cooled ion beams. The lack of observation of such crystalline ion beams is therefore in agreement with this tune condition, but whether the criterion is strictly correct or not remains untested. Information on this issue from beam emulation experiments in a table top size ion trap would, hence, be valuable before commissioning a large-scale, specialized storage ring for crystalline beams.

Today, Coulomb crystals can be produced routinely in experiments with laser-cooled ions in linear Paul traps (see, e.g., [9] and references therein). Up until now, radial confinement has been achieved by applying a sinusoidally timedependent rf voltage to the quadrupole rods of the trap. The choice of waveform for the time-varying rf voltage is, however, quite arbitrary. As pointed out in [10-12], a pulsed, square waveform would give rise to the same transverse dynamics for a charged particle as when traveling through the AG magnetic quadrupole lattice of a storage ring or a transport line [13]. This opens up the possibility of emulating storage-ring-like beam confinement in a trap, and in a recent

\footnotetext{
*Email address: niels.kjaergaard@ risoe.dk

${ }^{\dagger}$ Email address: krm@mic.dtu.dk

\#Email address: drewsen@ifa.au.dk
}

paper [12], we suggested to use this approach to test the stability of laser-cooled Coulomb crystals in an environment with AG strong focusing. It should be stressed that the confinement of such a pulse-excited trap is only storage-ringlike with respect to the focusing from discretely occurring quadrupole fields. In a real storage ring, bending from dipole fields is needed in order to make the beam circulate and a crystalline beam experiences shear at bend points [1]. In a simple trap setup this important effect will be absent, but on the other hand one has the possibility of isolating the crystalline stability problem regarding focusing.

In this Rapid Communication, we present results from such AG focusing experiments performed in Aarhus with a trapped ${ }^{24} \mathrm{Mg}^{+}$ion non-neutral plasma, laser cooled to such low temperatures (typically a few $\mathrm{mK}$ ) that spatial ordering of ions can be observed. We find that these Coulomb crystals become resonantly unstable when the plasma frequency exceeds half the rf drive frequency, which is equivalent to violating the tune condition for a storage ring.

The linear Paul trap employed in the experiments consists of four cylindrical electrodes of radius $R$ arranged in a quadrupole configuration as shown in Fig. 1(a). To emulate the discrete and finite focusing elements of an AG channel, rectangular voltage pulses are applied to achieve a time-varying voltage difference between adjacent rods with a waveform $\phi_{\tau}(t)$ of period $T=2 \pi / \omega_{\mathrm{rf}}$ as defined in Fig. 1(b). Denoting the inscribed radius of the interelectrode space by $r_{0}$, the resulting time-varying electric quadrupole potential is

$$
\Psi_{\rho}(x, y, t)=\frac{\phi_{\tau}(t)}{2} \frac{x^{2}-y^{2}}{r_{0}^{2}},
$$

corresponding to a regular so-called FODO lattice where the time elapsed between subsequent focusing and defocusing pulses is $T / 2$ and all pulses have the same duration $\tau T$ and amplitude $V_{\mathrm{rf}}$. For the present, simple choice of $\phi_{\tau}(t)$, the radial, pseudoharmonic confinement potential for charged particles arising from Eq. (1) has azimuthal symmetry and is characterized by a single, radial oscillation frequency $\omega_{\rho}$.

An axial confinement force is achieved by sectioning each of the quadrupole rods into three as shown in Fig. 1(a) and applying an additional dc voltage $U_{\mathrm{dc}}$ to the eight end pieces. 
(a)

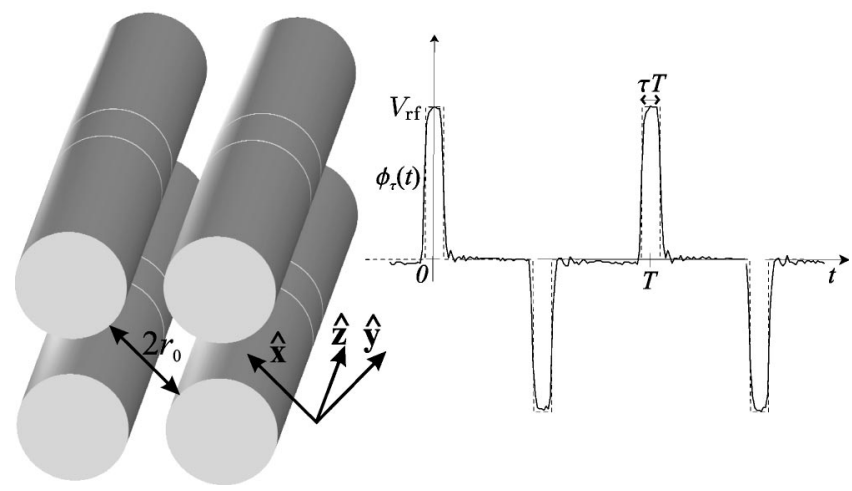

FIG. 1. (a) The linear Paul trap. Time-varying voltages are applied to the electrodes so that the resulting voltage difference between adjacent rods is given by $\phi_{\tau}(t)$ leading to radial confinement of ions. To obtain axial confinement, each electrode is sectioned into three and an additional dc voltage $U_{\mathrm{dc}}$ is applied to the eight end pieces with respect to the center pieces. (b) The pulsed, timevarying idealized (dashed line) and experimental (solid line) waveform $\phi_{\tau}(t)$.

This gives rise to a static, approximately harmonic potential $\Psi_{s}(x, y, z)=\eta z_{0}^{-2} U_{\mathrm{dc}}\left[z^{2}-\left(x^{2}+y^{2}\right) / 2\right]$, where $\eta$ is a geometric factor and $z_{0}$ is the length of the electrode center pieces. In terms of an energetic, charged particle beam, the harmonic axial confinement from $\Psi_{s}$ corresponds to the effective bunching restoring force within a bucket of a synchrotron. This restoring force can be derived from the discrete momentum kicks given by an rf bunching cavity [14]. We do not expect this approximation to have a crucial influence on the present investigations.

As is customary in the literature, we shall discuss the stability of radial motion in terms of the reduced parameters $q=2 e V_{\mathrm{rf}} / m r_{0}^{2} \omega_{\mathrm{rf}}^{2}$ and $a=-4 \eta e U_{\mathrm{dc}} / m z_{0}^{2} \omega_{\mathrm{rf}}^{2}$, where $e$ and $m$ are the charge and mass of the particles, respectively. A single-particle stability region can be derived as described in [12]. Only for $(q, a)$ parameters within the stability region is a particle radially bound to the trap. Coulomb crystals are known to have spheroidal envelopes in a harmonic trap [9]. Increasing $q$ through the rf waveform amplitude $V_{\text {rf }}$ leads to stronger radial confinement and more prolate (cigar shaped) crystals, whereas increasing the magnitude of $a$ through the end piece voltage $U_{\mathrm{dc}}$ leads to stronger axial confinement (accompanied by weaker radial confinement) and more oblate (pancake-shaped) crystals. This is the physical meaning of the dimensionless $q$ and $a$ parameters. It has been the object of the present experiments to determine the subset of the single-particle stability region for which Coulomb crystals are stable.

The total, effective confinement forces of the trap can be derived from the harmonic potential

$$
\Phi(\mathbf{r})=\frac{m}{2 e}\left(\omega_{z}^{2} z^{2}+\omega_{r}^{2} \rho^{2}\right),
$$

where $\omega_{z}=\sqrt{-a \omega_{\mathrm{rf}}^{2} / 2}$ is the single-particle axial oscillatory frequency, $\rho$ is the radial distance to the trap axis, and $\omega_{r}$ is

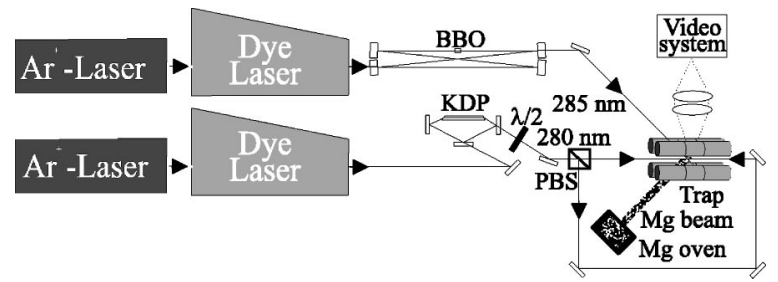

FIG. 2. Experimental setup. Cooling laser light $(\lambda=280 \mathrm{~nm})$ and photoionizing laser light $(\lambda=285 \mathrm{~nm})$ are derived from $\mathrm{Ar}^{+}$-laser pumped dye lasers by frequency doubling the outputs in nonlinear crystals (KDP and BBO, respectively) placed in external cavities. In the center of the trap, the photoionizing laser beam intersects an effusive, collimated $\mathrm{Mg}$ atomic beam at right angles. The cooling laser beam is split into two beams by a polarization beam splitter (PBS) and these two beams enter in counterpropagating directions along the trap axis. By rotating a half-wave plate $(\lambda / 2)$, the cooling forces can be balanced. The laser-induced fluorescence of Coulomb crystals is imaged by an intensified video system and the trapping parameters for which crystal melt can be recorded.

the effective, radial single-particle oscillation frequency. $\omega_{r}$ can be calculated using the transfer-matrix method of Courant and Snyder [13] as discussed in [12].

Neglecting variations on an interparticle scale, the density of a low-temperature, non-neutral plasma (cold fluid) in a harmonic trap is constant and given by

$$
n_{0}=\frac{\epsilon_{0}}{e} \nabla^{2} \Phi=\frac{\epsilon_{0} m}{e^{2}}\left(\omega_{z}^{2}+2 \omega_{r}^{2}\right)
$$

Bulk plasma oscillations in the cold fluid occur at the plasma frequency $\omega_{p}=\sqrt{e^{2} n_{0} / \epsilon_{0} m}$, which also equals the maximum bulk mode frequency in the case of a crystal [15]. From Eq. (3) we obtain $\omega_{p}^{2}=\omega_{z}^{2}+2 \omega_{r}^{2}$. To avoid a 1:2 (parametric resonance) relationship between the frequency of a bulk mode and the rf drive frequency, the criterion $2 \omega_{p}$ $<\omega_{\text {rf }}$ has to be fulfilled. This is equivalent to the aforementioned tune condition for a crystalline ion beam.

Figure 2 shows a schematic drawing of the experimental setup used to test the crystalline stability criterion. The trap has dimensions $R=4 \mathrm{~mm}, r_{0}=3.5 \mathrm{~mm}$, and $z_{0}=5.4 \mathrm{~mm}$ (corresponding to a geometric factor $\eta=0.992$ ) and is installed in a UHV chamber, where a pressure around $2.5 \times 10^{-10}$ torr is maintained. At the trap center, an atomic $\mathrm{Mg}$ beam is crossed at right angles by a light beam from a narrow bandwidth laser source and ${ }^{24} \mathrm{Mg}^{+}$ions are loaded into the trap by resonantly photoionizing ${ }^{24} \mathrm{Mg}$ via the $3 s^{2}{ }^{1} S_{0} \leftrightarrow 3 s 3 p^{1} P_{1}$ optical transition at $285 \mathrm{~nm}$ [16]. The ${ }^{24} \mathrm{Mg}^{+}$ions captured by the trapping fields are laser-cooled axially by two counterpropagating laser beams driving the $3 s^{2} S_{1 / 2} \leftrightarrow 3 p^{2} P_{3 / 2}$ closed optical transition at $280 \mathrm{~nm}$ with light from a tunable laser system. If the cooling rate is sufficiently high and the trap parameters $(q, a)$ are within the single-particle stability region, this may lead to the formation of Coulomb crystals with the ions arranged in spheroidal shells $[9,16]$. The light spontaneously emitted by the trapped ions during the laser-cooling cycle is recorded by an intensi- 


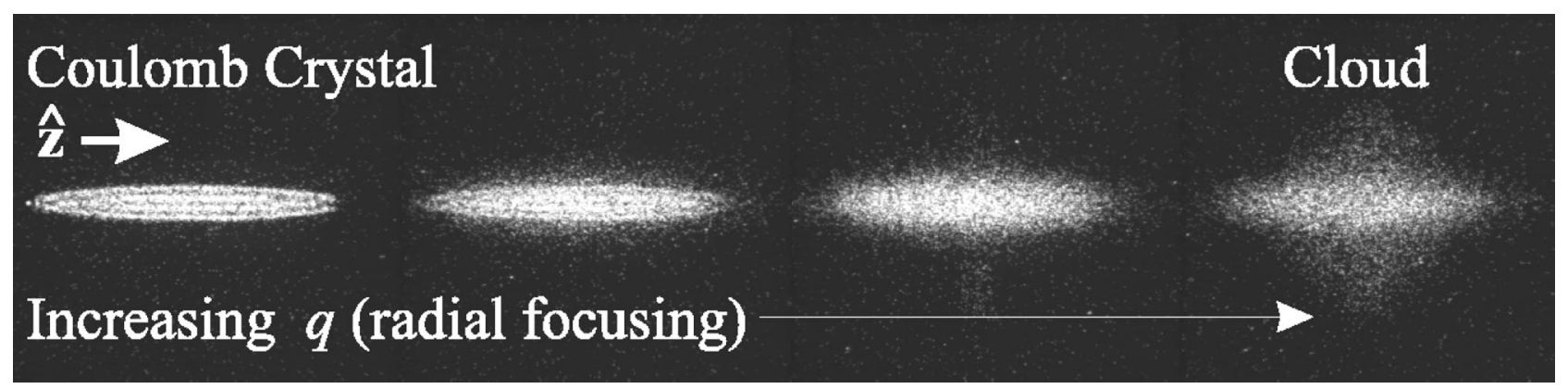

FIG. 3. A sequence acquired with the video system showing the melting process of a Coulomb crystal when the $q$ parameter of the trap is increased. $\hat{\mathbf{z}}$ indicates the axial direction of the trap.

fied digital camera system (see Fig. 3). The camera is read out at a rate of 5 frames/s and the direction of the optical axis through the trap center is $-(\hat{\mathbf{x}}+\hat{\mathbf{y}}) / \sqrt{2}$ [see Fig. 1(a)].

In a series of experiments, we have determined the subregion of the single-particle $(q, a)$ stability region where Coulomb crystals can be found to exist. Figure 4 shows the results in the case of $\omega_{\mathrm{rf}}=2 \pi \times 1 \mathrm{MHz}$ and $\tau=0.1$. The points represent parameters for which we directly observe Coulomb crystals to become unstable and melt (the crystals transform into a cloud state) while the double-hatched area is the parameter region for stable crystals. Each of these points is recorded by first forming a Coulomb crystal with trap parameters well within the double-hatched area and subsequently altering the parameters via $U_{\mathrm{dc}}$ or $V_{\mathrm{rf}}$ towards the low- $q$ or high- $q$ stability limits until melting occurs, as shown in Fig. 3. In this way we derive the boundaries of the double-hatched stability region for crystalline stability. For the present investigations the high- $q$ boundary is of dominant interest. The gray area beyond this boundary represents the part of the single-particle stability region for which

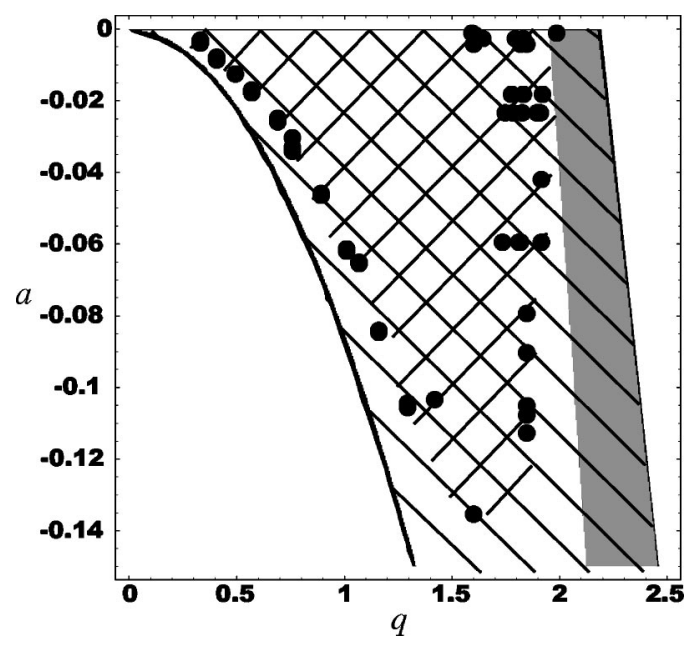

FIG. 4. For experiments with $\omega_{\mathrm{rf}}=2 \pi \times 1 \mathrm{MHz}$ and $\tau=0.1$, Coulomb crystals were observed to exist inside the /-hatched area which is a subset of the single-particle stability region (।-hatching). The gray area shows the part of the stability diagram for which $2 \omega_{p}>\omega_{\text {rf }}$. The trap $(q, a)$ parameters for which Coulomb crystals were observed to become unstable $(\bigcirc)$ comply reasonably well with the stability criterion $2 \omega_{p}<\omega_{\text {rf }}$.
$2 \omega_{p}>\omega_{\mathrm{rf}}$ and parametric resonances between the plasma bulk modes and the driving rf field are expected. Our results presented in Fig. 4 are in reasonable agreement with the theoretical prediction of parametric, resonant melting in the sense that Coulomb crystals cannot exist within this forbidden region. However, the stability limit as given by the data tends to constitute a more vertical line than expected, giving rise to some discrepancy for large negative values of $a$ where the crystals are less prolate. In a realistic storage ring this situation would not occur since the bunching restoring force is typically much weaker than the transverse confinement (i.e., $a$ would be very close to zero in terms of the trap) and the bunch shapes are very prolate. The data points in the high- $q$ limit were acquired by moving along iso- $a$ lines in the stability diagram, and although the reproducibility of melting points was generally quite good, a certain spread in the $q$ values for crystalline instability can be seen in Fig. 4 for some values of $a$, where consecutive measurements were performed.

Similar results to the ones presented in Fig. 4 were obtained in experiments with choices of $\tau$ in the range 0.070.14 and $\omega_{\text {rf }} / 2 \pi$ in the range $700 \mathrm{kHz}-2 \mathrm{MHz}$ [17]. Moreover, the results were independent of plasma size (50-1000 ions) although crystals containing only a few ions might traverse the stability limit. In the low- $q$ limit, crystals were observed to melt before reaching the single-particle stability limit. This may be attributed to the very weak radial confinement here, which leads to very oblate crystals with limited sympathetic cooling (via Coulomb interactions) of radial motion. Collisions with residual gas particles can hence more easily heat up the plasma.

In this Rapid Communication, we have exclusively focused on experiments with regular FODO lattices and a static, axial confinement force, but other more complex lattices can also be studied in our setup. We stress that the tune criterion that we have verified in a trap with storage-ring-like confinement is a necessary, but not sufficient, condition for attainment of Coulomb crystals. In a ring, additional complications arise, e.g., from shear due to bending of the crystal. The present setup has made it possible to address the stability problems regarding focusing separately. Notably, it seems that the stability criterion $2 \omega_{p}<\omega_{\text {rf }}$ should be taken very seriously when designing storage ring lattices for crystalline beam experiments. Beyond the stability limit we have, how- 
ever, observed crystals to reappear by rearranging themselves in an axially ordered structure with the ions situated in a string of disks [18]. These intriguing new structures, which will be the subject of a forthcoming paper, do not violate the stability criterion due to a decreased density and, hence, plasma frequency as compared to the corresponding unstable closed-shell structure.

In conclusion, we have investigated the stability of Coulomb crystals in a pulse-excited linear Paul trap that has the same transverse dynamics as storage rings with AG magnetic confinement. The observations agree well with current theory on crystalline plasmas and beams.

This work was supported by the Danish National Research Foundation through Aarhus Center of Atomic Physics (ACAP), the Danish Research Council (SNF), and the Carlsberg Foundation. We thank K. Støchkel for assistance during the experiments.
[1] Crystalline Beams and Related Issues, edited by D.M. Maletić and A.G. Ruggiero (World Scientific, Singapore, 1996).

[2] I. Lauer, U. Eisenbarth, M. Grieser, R. Grimm, P. Lenisa, V. Luger, T. Schätz, U. Schramm, D. Schwalm, and M. Weidemüller, Phys. Rev. Lett. 81, 2052 (1998).

[3] N. Madsen, P. Bowe, M. Drewsen, L.H. Hornekær, N. Kjærgaard, A. Labrador, J.S. Nielsen, J.P. Schiffer, P. Shi, and J.S. Hangst, Phys. Rev. Lett. 83, 4301 (1999).

[4] M. Steck, K. Beckert, H. Eickhoff, B. Franzke, F. Nolden, H. Reich, B. Schlitt, and T. Winkler, Phys. Rev. Lett. 77, 3803 (1996).

[5] H. Danared, A. Källberg, K.-G. Rensfelt, and A. Simonsson, Phys. Rev. Lett. 88, 174801 (2002).

[6] T. Schätz, U. Schramm, and D. Habs, Nature (London) 412, 717 (2001).

[7] X.-P. Li, A.M. Sessler, and J. Wei, in Proceedings of the Fourth European Particle Accelerator Conference (EPAC 94), edited by D. Dubin and D. Schneider (World Scientific, Singapore, 1994), Vol. 2, p. 1379.

[8] J. Wei, H. Okamoto, and A.M. Sessler, Phys. Rev. Lett. 80,
2606 (1998).

[9] L. Hornekær, N. Kjærgaard, A.M. Thommesen, and M. Drewsen, Phys. Rev. Lett. 86, 1994 (2001).

[10] H. Okamoto and H. Tanaka, Nucl. Instrum. Methods Phys. Res. A 437, 178 (1999).

[11] R.C. Davidson, H. Qin, and G. Shvets, Phys. Plasmas 7, 1020 (2000).

[12] N. Kjærgaard and M. Drewsen, Phys. Plasmas 8, 1371 (2001).

[13] E.D. Courant and H.S. Snyder, Ann. Phys. (N.Y.) 3, 1 (1958).

[14] M. Reiser, Theory and Design of Charged Particle Beams (Wiley, New York, 1994).

[15] D.H.E. Dubin and J.P. Schiffer, Phys. Rev. E 53, 5249 (1996).

[16] N. Kjærgaard, L. Hornekær, A.M. Thommesen, Z. Videsen, and M. Drewsen, Appl. Phys. B: Lasers Opt. 71, 207 (2000).

[17] We note that these ranges of parameters are comparable to what one might have in a realistic storage ring.

[18] N. Kjærgaard, K. Mølhave, and M. Drewsen, in Non-Neutral Plasma Physics IV, edited by F. Anderegg, C. Fred Driscoll, and L. Schweikhard, AIP Conf. Proc. No. 606 (AIP, Melville, NY, 2002), p. 145. 